\title{
Biomechanical aspects of catheter-related thrombophlebitis
}

\author{
Oren Moshe Rotman*, Dalit Shav, Sagi Raz, Uri Zaretsky, Shmuel Einav
}

Department of Biomedical Engineering, Tel Aviv University, Tel Aviv, Israel

Email: *orenrotm@post.tau.ac.il

Received 30 September 2013; revised 29 October 2013; accepted 12 November 2013

Copyright (C) 2013 Oren Moshe Rotman et al. This is an open access article distributed under the Creative Commons Attribution License, which permits unrestricted use, distribution, and reproduction in any medium, provided the original work is properly cited.

\begin{abstract}
Short peripheral catheters (SPCs) are the most common intravenous devices used in medical practice. Short peripheral catheter thrombophlebitis (SPCT) is the most frequent complication associated with SPCs, causing discomfort and usually leading to removal of the catheter and insertion of a new one at a different site. The aim of this research was to explore whether biomechanical factors, in addition to biochemical factors, also play a role in the formation of thrombophlebitis. Hence, two of the biomechanical aspects of SPCT were investigated: the physical pressure load exerted by the SPC on the endothelial monolayer, and disturbances in the flow patterns due to the SPC. Endothelial activation was studied by subjecting human umbilical vein endothelial cells (HUVEC) to a weight load of SPC pieces and measuring the release profile of von-Willebrand Factor (vWF) over time, using ELISA. vWF release was chosen as the measure for endothelial activation since it was the major component of the Weibel-Palade Bodies (WPBs), which underwent exocytosis by endothelial cells during activation. Flow patterns were analyzed on a 3D computational fluid dynamics (CFD) model of a brachiocephalic vein with SPC. vWF release profiles were significantly higher in the HUVECs subjected to the load, indicating HUVEC activation. CFD simulations demonstrated a decrease in flow velocities along the catheter body, between the catheter and the vein, due to an enlarged boundary layer. Results indicate that the contact region between the SPC body and the vein wall can be partially responsible for SPCT development, and inflammatory and coagulatory processes initiated by stimulated endothelial cells may be amplified due to disturbed blood flow.
\end{abstract}

Keywords: Endothelial Activation; vWF;

${ }^{*}$ Corresponding author.
Thrombophlebitis; Phlebitis; Short Peripheral Catheters; Infusion; Intravenous Access; CFD

\section{INTRODUCTION}

Short peripheral catheters (SPC) are the most widely used intravenous devices in medical practice today, particularly in hospitals and intensive care units. The SPC is commonly inserted into veins of the upper extremities to administer fluids, medications and blood products, or for prophylaxis before procedures. Short peripheral catheter thrombophlebitis (SPCT) is the most frequent complication associated with SPCs, with prevalence in hospitalized patients ranging from $2.6 \%$ [1] to $77.5 \%$ [2]. This inflammatory process of the vein wall is characterized by pain, tenderness, warmth, erythema, swelling, and sometimes palpable thrombosis of the cannulated vein. In the past, SPCT was thought to be initiated by infection from the insertion site, but studies of catheter tip cultures suggest it may be mediated or initiated by a noninfectious inflammatory process [1,3-6]. Moreover, SPCT symptoms such as local swelling and erythema on the skin surface commonly appear along the venous track [7], making the catheter penetration wound not necessarily initiate the inflammatory process. These findings led us to hypothesize that the inflammatory process of SPCT is initiated by the interaction between the catheter body, the vein wall, and the blood flow.

SPCT causes discomfort and usually results in removal of the catheter and insertion of a new one at a different site. Repeated episodes can lead to venous access difficulties and more invasive procedures, such as central venous catheter placement. This usually results in delayed administration of parenteral medications, lengthened hospital stay and increased costs [6]. Several mechanisms of SPCT pathogenesis have been suggested, including vein wall injury combined with stasis and inflammation that lead to thrombosis [8], and sterile inflammation caused by chemical irritation of the endothe- 
lium due to the infusate or catheter material [9-11]. Despite the numerous studies, the pathogenesis of SPCT remains unclear.

Endothelial cells, which act as the barrier between blood and organ tissues, have the capability to regulate local inflammation and coagulation reactions. Where the immediate response is needed, such as at sites of vascular injury, the endothelial cells are activated and support local recruitment of leukocytes and platelets. This recruitment is mediated by the release of the contents of Weibel-Palade Bodies (WPBs) [12]. While a variety of chemical stimulations for WPBs exocytosis are reported in the literature, information is sparse on the effect of physical pressure of a foreign body, such as that exerted by SPCs on endothelial cells.

Flow disturbances are known to affect platelet activation and coagulation by accumulation of shear stresses in recirculation regions and jets [13], and in stagnation regions due to insufficient flush of highly potent coagulation substances. Such flow regimens may exist in the vicinity of the SPC and participate in the inflammatory and coagulatory processes. To the best of our knowledge, there are no reports on flow patterns in the context of SPCT.

The present study is a pioneering work that evaluates the possible effect of two biomechanical aspects of SPCT development: 1) physical pressure of the SPC on the vein wall that irritates and activates the endothelial cells, and 2) flow disturbances in the vicinity of the catheter.

\section{MATERIALS AND METHODS}

The effect of physical pressure load of SPCs on vein walls was studied in vitro in cultured endothelial cells. Flow patterns in a cannulated vein model were examined using computational flow dynamics (CFD) simulations.

\subsection{In Vitro Experiments}

Fluorinated ethylene propylene (FEP) 16 gauge $(1.3 \mathrm{~mm}$ inner diameter, $1.7 \mathrm{~mm}$ outer diameter) short peripheral infusion catheters (Ven-O-Lit $\left.{ }^{\circledR}\right)$ were placed over human umbilical vein endothelial cells (HUVEC). Chemical reactions of the cells to the catheter material were avoided by employing only genuine infusion catheter material. The catheters were cut into $1 \mathrm{~cm}$ long pieces, into which $0.7 \mathrm{~cm}$ long stainless steel pins were inserted to prevent the catheter pieces from floating in the medium (Figure 1), forming weights of approximately 75 $\mathrm{mg}$ each. This weight was chosen after banding similar SPC units on digital scales for up to $30^{\circ}$, an angle that represents the entrance orientation of the SPC into the vein. Each of these weights exerted a physical pressure load of approximately $67 \mathrm{~Pa}(0.5 \mathrm{mmHg})$, estimated by dividing the weight by the contact surface area. Fourteen

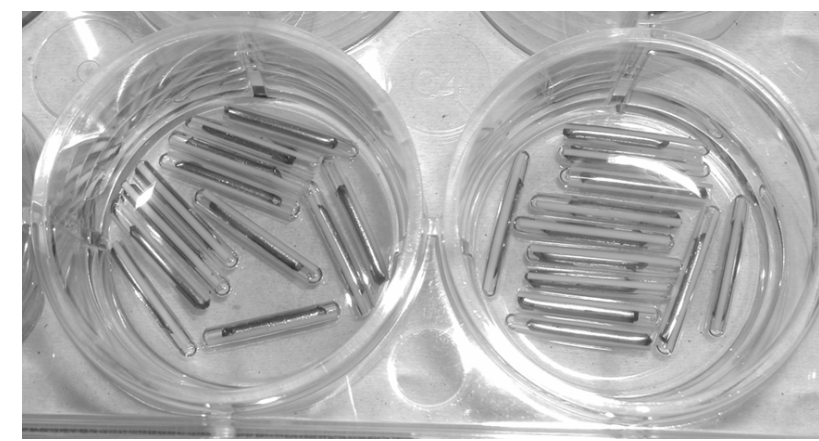

Figure 1. Catheter and stainless steel pieces forming load on HUVEC in a 12 -well plate.

weights were then placed over a monolayer of HUVEC in each well of a 12-well plate, in a static flow environment, with a confluency of more than $95 \%$. The HUVEC samples were divided into two groups of 3 samples each: the test group, which was subjected to the weight load, and a control group. The two groups were sampled simultaneously. For statistical purposes, the in vitro protocol was repeated twice in a non-dependent fashion, each time using a different cell passage. HUVEC activation was measured by quantification of von-Willebrand Factor (vWF) release, which is the major component of the WPBs and responsible for platelet adhesion to stimulated endothelial cells [12]. Increased vWF release over time compared with the control group will indicate WPB exocytosis-evidence of the increased HUVEC activetion that promotes both inflammatory and coagulatory responses. vWF release was chosen over other components of the WPBs because it is abundant and therefore easy to detect.

\subsubsection{Cell Culture}

HUVEC (Lonza) were grown in MCBD-131 medium supplemented with $5 \%$ foetal bovine serum (FBS), $2 \mathrm{mM}$ L-glutamine, $100 \mathrm{U} / \mathrm{ml}$ penicillin, and $0.1 \mathrm{mg} / \mathrm{ml} \mathrm{strep-}$ tomycin (Biological Industries Beit Haemek, Israel), 2 $\mathrm{ng} / \mathrm{ml}$ insulin, $0.5 \mathrm{ng} / \mathrm{ml}$ epithelial growth factor (Sigma Aldrich), $2 \mathrm{ng} / \mathrm{ml}$ basic-fibroblasts growth factor (bFGF) (PeproTech, Inc., USA) and $1 \mu \mathrm{g} / \mathrm{ml}$ hydrocortisone (Sigma Aldrich) cultured at $37^{\circ} \mathrm{C}, 5 \% \mathrm{CO}_{2}$, in a humidifier incubator. Cells from passages 5 - 10 were used. Cell morphology and confluence were inspected with a Nikon eclipse TS100 phase inverted microscope and captured with a Nikon Coolpix 4500 digital camera.

\subsection{2. vWF Measurement}

Cells were seeded at near confluence density of 20,000 cells $/ \mathrm{cm}^{2}$ on a $0.1 \%$ gelatin (Sigma Aldrich) coated 12well plate. When the cells reached $100 \%$ confluence, the medium was replaced and the weights were placed on the cells. Medium samples were collected and kept at $-20^{\circ} \mathrm{C}$ until analyzed. During incubation the cells were kept in a 
humidifier incubator at $37^{\circ} \mathrm{C}$ and $5 \% \mathrm{CO}_{2}$. Samples were taken from the wells of both groups at four different times: at the beginning of the experiment $(t=0)$, and after 20, 40 and 60 minutes. Sixty minutes was found to be sufficient for this setup after several trials with different time scales, as elaborated in the Discussion section. Quantification of vWF was done using Assay-Max ELISA kit (AssayPro). Briefly, samples were incubated for 2 hours using a 96-well microplate coated with murine monoclonal antibody against $\mathrm{vWF}$, after which the samples were removed and the wells were incubated for 60 minutes with biotinylated vWF antibody for another 30 minutes with streptavidin-peroxidase conjugate. The plate was then incubated for 10 minutes with a stabilized peroxidase chromogen substrate, after which a stop solution (containing $0.5 \mathrm{~N}$ hydrochloric acid) was added to the chromogen solution. The plate was read at $450 \mathrm{~nm}$ by a spectrophotometer (SpectraMax 340PC384, Molecular Devices Corp., Sunnyvale CA, USA). All incubations were performed at $37^{\circ} \mathrm{C}$. The wells were washed 5 times between each step using the wash buffer supplied in the kit. For each procedure, a calibration curve was calculated using a human vWF standard protein provided in the kit.

\subsubsection{Statistical Analysis}

Statistical analysis was performed by ANOVA with repeated measures (over time) with covariance structure of compound symmetry, using SPSS software (SPSS Inc.), and using the vWF concentration results as input. Significance level was considered as $\mathrm{p}<0.05$.

\subsection{Numerical Simulations}

\subsubsection{Governing Equations}

The equations governing continuity (Eq.1) and momentum (Eq.2) for incompressible, viscous and laminar blood flow were:

$$
\begin{gathered}
\nabla \cdot U=0 \\
(U \cdot \nabla) U=-\frac{1}{\rho} \nabla P+v \nabla^{2} U
\end{gathered}
$$

where $U$ is the velocity vector, $P$ is the static pressure, $\rho$ is the fluid density, and $v$ is the kinematic viscosity.

\subsubsection{Boundary Conditions}

Cephalic vein flow rate is normally $28 \mathrm{ml} / \mathrm{min}$ for a vein diameter of $2.3 \mathrm{~mm}$ [14]. In order to keep the Reynolds number (Re) in our enlarged model of $5 \mathrm{~mm}$ diameter similar to its physiological value, the flow rate was adjusted to $60 \mathrm{ml} / \mathrm{min}(\operatorname{Re}=73)$. The effect of flow of three additional flow rates was examined: $28 \mathrm{ml} / \mathrm{min}(\mathrm{Re}=34)$, $45 \mathrm{ml} / \mathrm{min}(\operatorname{Re}=55)$, and $75 \mathrm{ml} / \mathrm{min}(\operatorname{Re}=91)$. The flow rates were applied to the vein by setting a constant average velocity at the vein inlet $(2.38 \mathrm{~cm} / \mathrm{s}, 3.87 \mathrm{~cm} / \mathrm{s}, 5.1$ $\mathrm{cm} / \mathrm{s}$, and $6.38 \mathrm{~cm} / \mathrm{s}$ for flow of $28 \mathrm{ml} / \mathrm{min}, 45 \mathrm{ml} / \mathrm{min}$, $60 \mathrm{ml} / \mathrm{min}$, and $75 \mathrm{ml} / \mathrm{min}$, respectively). Volume outflow condition was set at the vein outlet, meaning that all the volume of flow in the model was drained through the outlet surface. Symmetry condition was applied to the model symmetry plane, such that the velocity gradients across this plane were equal to zero. No slip condition was defined at any of the interfaces between the blood and the catheter or vein walls. The fluid properties were set as whole blood, with density of $\rho=1060 \mathrm{Kg} / \mathrm{m}^{3}$, and dynamic viscosity of $\mu=3.5 \mathrm{mPa} \cdot \mathrm{s}$.

\subsubsection{Computational Method}

The governing equations (Eqs.1 and 2) were simultaneously solved by the computational fluid dynamics package of Fluent (Fluent Inc., Lebanon, NH, USA). The implicit solver formulation was used for solving the partial differential equations in a segregated manner. A spatially second-order upwind discretization scheme was used to minimize numerical dissipation.

\subsubsection{Assumptions}

The numerical model was based on the following assumptions: (A) steady flow rate $[15,16]$; (B) rigid vein. Veins, which are normally considered collapsible tubes, can be assumed to be rigid when fully inflated. Such is the case with the cephalic vein while the arm is below the level of the heart [17]; (C) the flow is laminar and the fluid is Newtonian and incompressible; (D) the catheter is sealed at its non-invasive end, and is placed inside the vein without injection or withdrawal of fluids through it. This was done because for most of the time the SPC is in-situ, it is actually not in use and SPCT still develops.

\subsubsection{Geometry and Computational Meshing}

The computational model geometry was that of a 3D cannulated cephalic vein (Figure 2). The 3D geometry and the meshing were designed using GAMBIT (Fluent Inc.). While the cephalic vein diameter usually ranges from $1.9-3.9 \mathrm{~mm}$, reaching from $1 \mathrm{~mm}$ to over $6 \mathrm{~mm}$ in actual measurements $[14,18,19]$, we chose a $5 \mathrm{~mm}$ inner diameter and $85 \mathrm{~mm}$ long straight tube section to make it compatible with the in vitro model in our laboratory. A 16 gauge ( $1.3 \mathrm{~mm}$ inner diameter, $1.7 \mathrm{~mm}$ outer diameter) catheter was placed inside the vein with an entrance orientation of $30^{\circ}$ relative to the vein symmetry axis. The catheter length from its contact with the vein was defined as $25 \mathrm{~mm}$, and was set to be sunk $0.25 \mathrm{~mm}$ into the vein wall and underlying tissue to simplify the meshing in the contact area. The vein length upstream to the catheter insertion site was extended by $23 \mathrm{~mm}$ to allow the flow to develop before it reached the catheter. The catheter lumen length was set for $10 \mathrm{~mm}$ from the catheter tip backwards. The model was cut along its plane of symmetry 


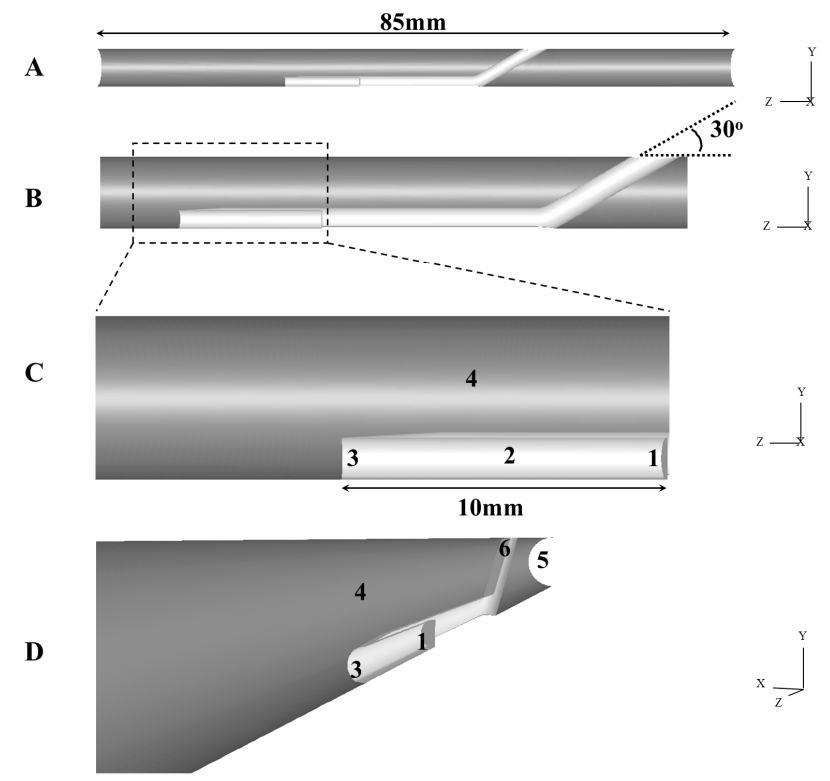

Figure 2. The computational model geometry: (A) side view of the entire geometry, $(\mathrm{B})$ side view zooming in on the catheter inside the vein, (C) further zooming in on the catheter lumen and tip, and (D) diagonal view of the model. Legend: (1) catheter entrance, (2) catheter lumen, (3) catheter tip, (4) vein lumen, (5) vein entrance, and (6) catheter insertion site.

(plane YZ) to save computational time.

The 3D geometry was converted with GAMBIT (Fluent Inc.) into discrete mesh using 1,010,690 hexahedral and tetrahedral elements. Maximum element size was $8.75 \times 10^{-3} \mathrm{~mm}^{3}$, and the total volume of the computational model was $805.29 \mathrm{~mm}^{3}$.

Mesh convergence study was performed to ensure that the solution in each case differed by no more than $1 \%$ in the velocity values.

\section{RESULTS}

\section{1. vWF Concentrations}

The data are summarized in Figure 3. In experiment repetition number 2 , the concentrations of samples Control $1-3$ and Test $2-3$ at $\mathrm{t}=0$ were manually set to 0 $\mathrm{mU} / \mathrm{ml}$, since the values achieved by the calibration curve were negative and close to zero. The data of Control 3 and Test 3 for $t=40$ minutes in experiment repetition 2 were discarded from the statistical analysis due to inappropriate manipulation of the relevant samples. The elevation in vWF concentration in the medium over time was considerably higher for most samples of the test group compared with the corresponding samples of the control group. The overall time course was significant ( $p$ $<0.001$ ) in both the control and test groups. The interaction between time course and experimental repetition was not significant $(\mathrm{p}=0.986)$. Most importantly, the interaction between time course and groups was signifi- cant $(p=0.013)$, evidenced by the increased release of $\mathrm{vWF}$ in the test group. The averages of the time steps also differed significantly between the control and test groups $(\mathrm{p}=0.03)$.

\subsection{Cell Morphology}

Cells in the control group (Figure 4) showed no differences between $t=0$ and $t=60$ in cell alignment and confluence density: at both time points cell confluence remained almost $100 \%$ with no signs of cell mitosis or stress, and the cells remained stretched with no preferred orientation in the field of view. In contrast, the test group cells exhibited significant changes between $t=0$ and $t=$ 60: their number had decreased, resulting in cell-free areas throughout the field of view; some of the remaining ones had assumed a round shape, suggesting they were about to detach, and several had become less smooth, denoting stress. In addition, the medium contained a substantial amount of debris (not seen in the images).

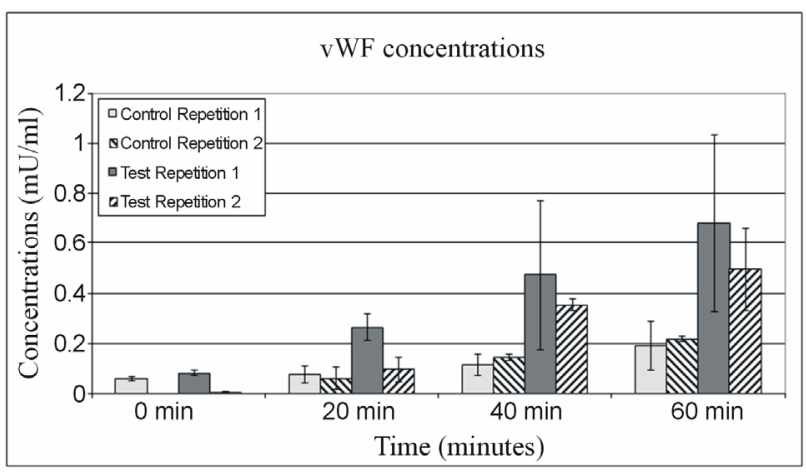

Figure 3. Graphic representation of vWF concentrations in the medium, measured by ELISA. The concentrations are presented as mean \pm standard deviation.

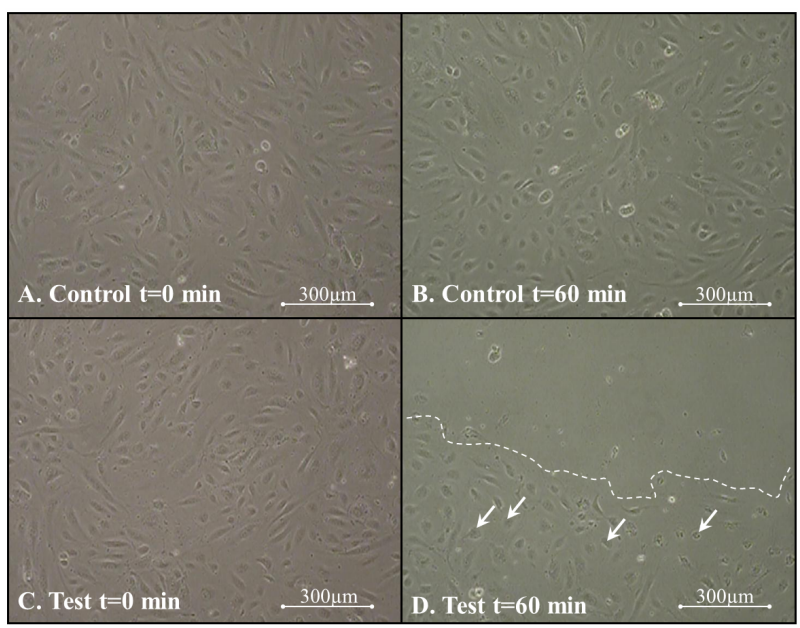

Figure 4. Images of HUVEC taken at time points $t=0$ minutes $((\mathrm{A})$ and $(\mathrm{C}))$ and $\mathrm{t}=60$ minutes ((B) and (D)). The white dashed lines mark areas clear of cells. The white arrows point to round shaped cells. 


\subsection{CFD Simulation}

The fluid path-lines smoothly followed the catheter outer surface at the insertion site, in the region where the catheter met the opposite wall of the vein, and along the catheter body. The catheter insertion site was free of flow disturbances and there was no apparent effect on the flow patterns downstream along the catheter wall. Two untoward phenomena were noticed: 1) As the flow passed the catheter tip (Figure 5), a small recirculation region appeared partially inside the catheter lumen. The recirculation region was $1.7 \mathrm{~mm}$ long in the $60 \mathrm{ml} / \mathrm{min}$ flow rate case, with no significant difference at the other examined rates (range $1.64 \mathrm{~mm}$ to $1.82 \mathrm{~mm}$ ). The surface area in which there were WSS below $0.1 \mathrm{~Pa}$ grew larger as the flow decreased. Goel and Diamond [18] showed that the maximum WSS at which red blood cells adhere to platelets, neutrophils, and polymerized fibrin - adhesion that supports increased thrombus formation-is $0.1 \mathrm{~Pa}$. The WSSs in the vein model without the catheter were 0.135 $\mathrm{Pa}, 0.23 \mathrm{~Pa}, 0.30 \mathrm{~Pa}$, and $0.38 \mathrm{~Pa}$ for flow rates of 28 $\mathrm{ml} / \mathrm{min}, 45 \mathrm{ml} / \mathrm{min}, 60 \mathrm{ml} / \mathrm{min}$, and $75 \mathrm{ml} / \mathrm{min}$, respectively. (2) The second phenomenon was a significant decrease in the flow velocity and WSS (Figure 6) on both sides of the catheter (along the $\mathrm{x}$ axis) as a result of an enlarged boundary layer. The mean axial velocities of the profiles with the catheter (Figure 7) were reduced on average by a factor of 2.76 due to the catheter. In contrast, maximal axial velocities (along the y axis) increased in the center of the vein lumen due to the catheter: increases of $0.81 \mathrm{~cm} / \mathrm{s}, 1.18 \mathrm{~cm} / \mathrm{s}, 1.39 \mathrm{~cm} / \mathrm{s}$, and $1.53 \mathrm{~cm} / \mathrm{s}$ for the flow rates of $28 \mathrm{ml} / \mathrm{min}$ to $75 \mathrm{ml} / \mathrm{min}$. This, however, did not significantly affect the vein maximal WSS on the y-axis $(0.2 \mathrm{~Pa}, 0.31 \mathrm{~Pa}, 0.41 \mathrm{~Pa}$, and $0.51 \mathrm{~Pa}$ for flow rates of $28 \mathrm{ml} / \mathrm{min}, 45 \mathrm{ml} / \mathrm{min}, 60$ $\mathrm{ml} / \mathrm{min}$, and $75 \mathrm{ml} / \mathrm{min}$, respectively).

\section{DISCUSSION}

The elevated concentrations of vWF in the test group indicate that the HUVEC was stimulated by the catheter weights. The slight rise in vWF over time in the control group can be accounted for by the known normal secretion of small amounts of vWF by endothelial cells [19]. This is supported by the absence of change in cell shape and confluence in this group (Figure 4). The large difference in VWF concentration between experiment repetitions 1 and 2 can be attributed to the fact that each of the repetitions was performed independently on cells from a different passage [20]. Despite the wide variation, the vWF release profile of the test group differed significantly over time from that of the control group in both independent repetitions. The higher release profiles reflected in the mean concentrations suggest ongoing stimulus of the test group cells.

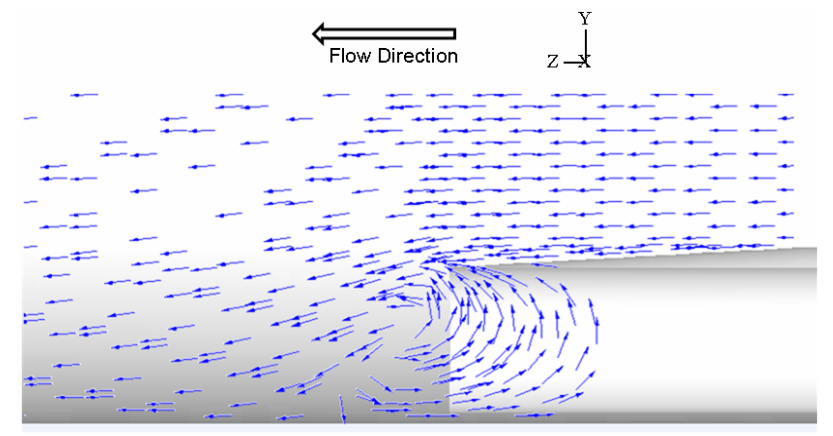

Figure 5. Side view of the catheter tip region with vectors describing the path-lines of flow, indicating a recirculation region at the catheter tip.

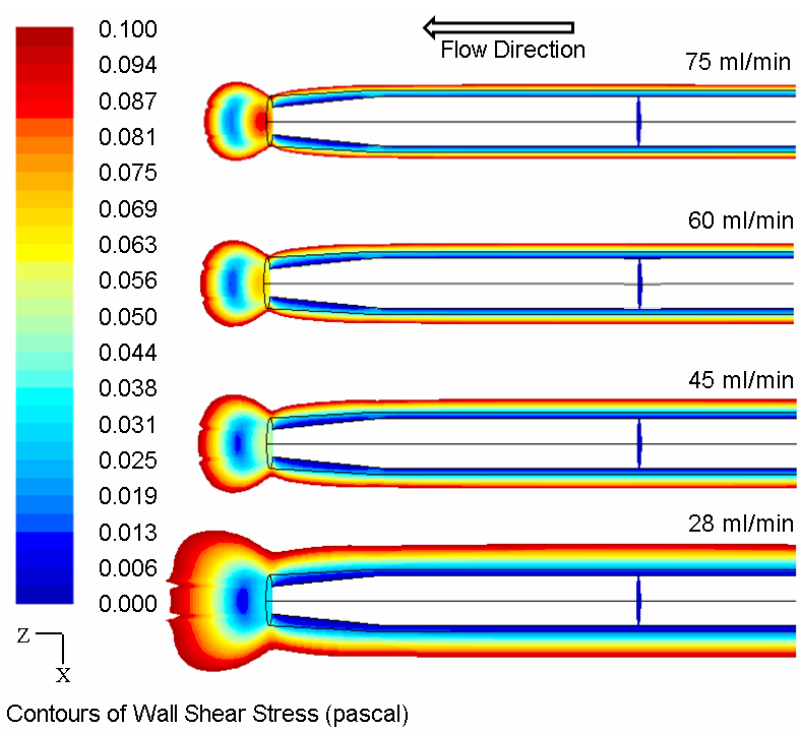

Figure 6. Top view of the vein WSSs in regions surrounding the contact area between the catheter and the vein. The WSSs are presented with an upper limit of $0.1 \mathrm{~Pa}$.

Unlike sustained hydrostatic pressure, the catheter is a physical impediment to metabolite transport processes between the culture layer and the nutrient medium. While sustained hydrostatic pressure of upto $7 \mathrm{mmHg}$ stimulates HUVEC proliferation [21], a relatively low physical pressure of less than $1 \mathrm{mmHg}$ in our study caused cell activation and death. The bald areas clearly seen in Figure 4(D) are those of the greatest physical load and most probably the contact areas between the cells and the catheter pieces. Moreover, in areas where the cells remained dense, some of them changed shape from elongated and stretched to round, indicating a disturbance or stress.

The mean axial velocities in proximity to the vein wall (on the $\mathrm{x}$-axis), obtained by CFD analysis, were reduced on average by a factor of 2.76 when the catheter was placed. The WSSs in this region were also reduced, creating a surface area surrounding the catheter of WSSs below $0.1 \mathrm{~Pa}$ (Figure 6). This reduction was caused by 

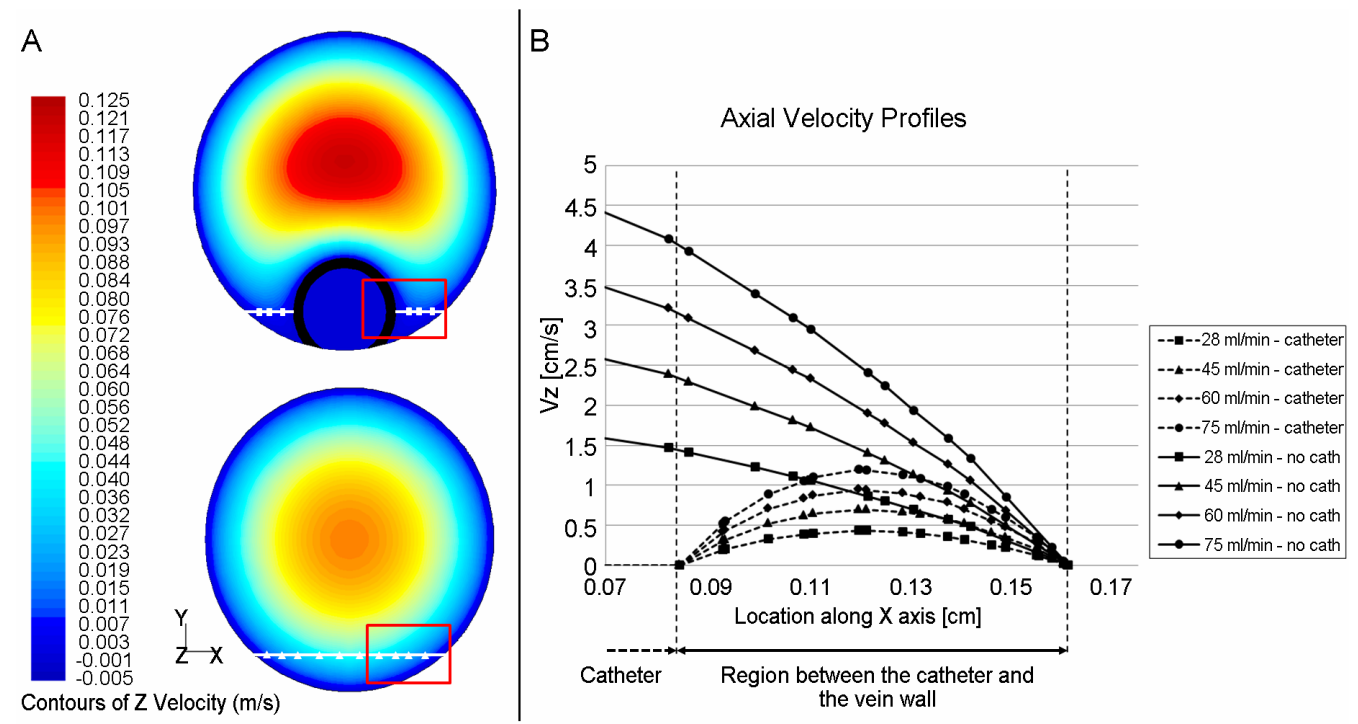

Figure 7. (A) Front view of the axial velocity contours at $60 \mathrm{ml} / \mathrm{min}$ vein flow: Top-vein with a catheter. Bottom - vein without a catheter. The horizontal white lines with rectangles (vein with a catheter) and triangles (vein without a catheter) are the lines along which velocity profiles were sampled for comparison. (B) Axial velocity profiles for the different vein flow rates examined, with and without a catheter, focusing on the region between the catheter and the vein wall on the x-axis (marked in A by a red rectangle).

an enlarged boundary layer between the vein wall and the catheter body. It was expected that the lower WSSs themselves would not activate the endothelial cells, as the WSSs in veins were naturally very low. Nor did the presence of the catheter have a marked effect on the WSSs. We believe that the major effect of the reduced velocities and WSS was to create a supportive environment for adhesion of rolling leukocytes and platelets to activated endothelium, and to each other, promoting a local inflammatory process in the vein wall and thrombosis formation in the lumen [12]. This mechanism is supported by Goel and Diamond's work [18] showing that a WSS below $0.1 \mathrm{~Pa}$ enables the circulating red blood cells to adhere to platelets, neutrophils, and polymerized fibrin, resulting in thrombus growth. The in vitro results confirmed our hypothesis that the endothelial cells would be activated in the precise location of contact between the catheter and the vein wall, the same location where we observed that significantly reduced flow velocities and reduced WSSs. While our hypothesis appears to have been proven, it needs confirmation from studies of endothelial activation under direct flow conditions.

Another important flow disturbance was the recirculation region that appeared at the catheter tip, partially inside the catheter lumen. A recirculation region in such a location can amplify local inflammatory and coagulatory processes, and slowly dilute the catheter lumen with blood products that may clot and eventually block the lumen and render it non-functional.

According to the CFD results, the flow rate did not seem to have a significant effect on the flow field, as the flow patterns for all simulated rates smoothly followed the catheter outer surface from insertion site to catheter tip. Nor did the size of the recirculation region at the catheter tip appear to be affected by the flow rate. The reduced flow rate did, however, slightly increase the region of very low WSSs around the catheter. It might be that low flow rates create a larger surface area with the potential to promote the rapid thrombus formation.

The findings of this research support the hypothesis that biomechanical factors participate in SPCT pathogenesis, and explain the mechanisms of the common complication associated with peripheral catheters. Most of the improvements in SPCs in recent years consisted of changes in the polymeric material of the cannula. Our results point to the need for geometric modifications to the SPC that will eliminate the contact between the catheter body and the vein wall and avoid the formation of flow stagnation and recirculation regions. Two such geometric modifications are illustrated in Figure 8: significant shortening of the SPC to avoid contact between the cannula and the opposite vein wall, and a protrusion on the cannula outer surface as an anchor to prevent the cannula from slipping outside the vein.

The biomechanical mechanism suggested by the results of the numerical simulations and in-vitro experiments conducted in this study involves prolonged irritation and activation of the endothelial cells by the SPC due to physical contact between them, and flow disturbances caused by the catheter that promoted local inflammatory and coagulatory processes. These flow patterns most likely support, rather than initiate, local reactions.

As a pioneering work, this study has limitations that 


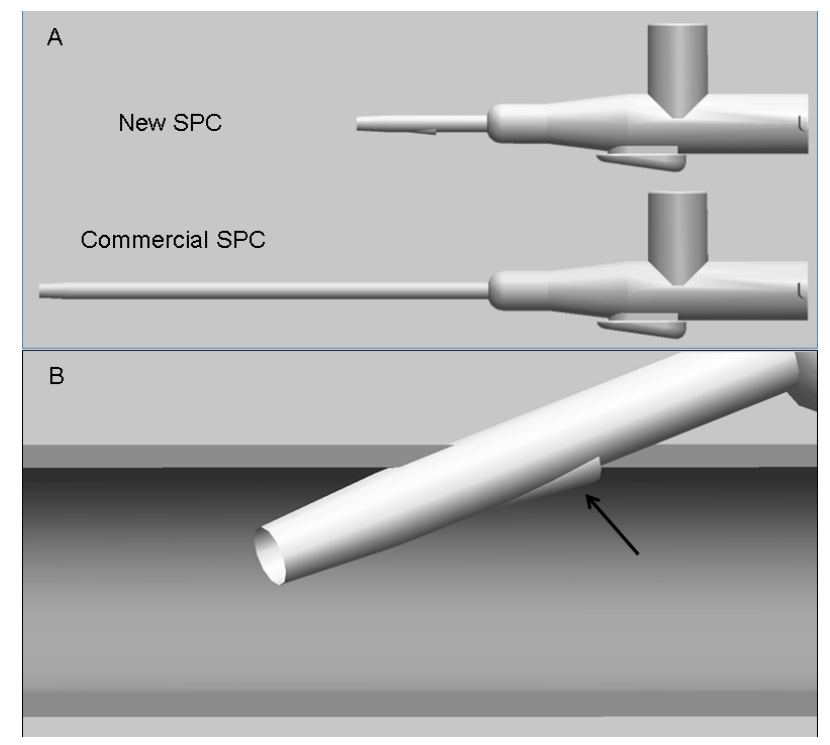

Figure 8. Example of an SPC with geometric modifications. (A) Comparison of the suggested SPC and a commercial SPC. (B) Illustration of the suggested SPC positioned in a vein. The arrow points to the protrusion on the catheter outer surface.

will need to be addressed in future research. The most serious one is that the in vitro experiments and the numerical simulations were conducted separately. Because incorporating a flow system into the HUVEC experimental setup would have diluted the vWF concentrations to an undetectable level, we chose at this stage to investigate the flow and the catheter-HUVEC interaction separately. We are planning a second generation system that will link the simulations to the experiments in a more robust manner. Another limitation is the difference between the time scale simulated in vitro with the HUVEC and the time scale for SPCT in vivo. The 60-minute period chosen for the in vitro experiment was found to be sufficient after several trials with different scales. While in clinical practice the time scale for SPCT is approximately 3 days [22], our choice of 60 minutes is justified on a number of fronts. As mentioned above, bald areas free of cells remained in the culture at 60 minutes, after which the cell numbers were no longer comparable and there was no reason to continue the experiment. The time scale is affected by factors other than the tested biomechanical ones: for example, the in vitro test had no flow, which meant that active substances secreted by the cells over time did not flush away. The absence of this process amplified the endothelial activation and made the time scale shorter. Previous works that investigated vWF secretion by chemical stimuli on cultured endothelial cells also used a 60-minute time scale [19,23]. Michaux et al. [19] reported that after 1 hour of chemical stimuli (by Calcium ionophore A23187 and by phorbol ester PMA), the HUVEC had secreted about $40 \%$ of their total vWF. Another limitation of the study was that irritation of the vein wall by the catheter tip and possible mechanical injury during the catheter insertion were not investigated. Such injuries to the vein wall could expedite local inflammatory and coagulatory reactions. We chose to focus on more "chronic" biomechanical factors that take place while the SPC is in-situ, and not on factors that can be avoided by better manipulation by the medical staff.

Despite of the limitations, this is the first study to investigate biomechanical aspects of SPCT. The results support our hypothesis and lay the groundwork for more comprehensive investigations that will examine the effect of different pressure loads on HUVEC activation, the incorporation of flow in the in vitro experiments, and the simulation of the bending of the SPC on the vein wall. Such studies will enhance our understanding of the effects of load on endothelial cells.

\section{ACKNOWLEDGEMENTS}

The study was partially supported by the Drown Foundation and the Berman Fund.

\section{REFERENCES}

[1] Malach, T., Jerassy, Z., Rudensky, B., et al. (2006) Prospective surveillance of phlebitis associated with peripheral intravenous catheters. American Journal of Infection Control, 34, 308-312. http://dx.doi.org/10.1016/j.ajic.2005.10.002

[2] Lundgren, A., Wahren, L.K. and Ek, A.C. (1996) Peripheral intravenous lines: Time in situ related to complications. Journal of intravenous nursing, 19, 229-238.

[3] Woodhouse, C.R. (1980) Infusion thrombophlebitis: The histological and clinical features. Annals of The Royal College of Surgeons of England, 62, 364-368.

[4] Cornely, O.A., Bethe, U., Pauls, R. and Waldschmidt, D. (2002) Peripheral Teflon catheters: Factors determining incidence of phlebitis and duration of cannulation. Infection Control and Hospital Epidemiology, 23, 249-253. http://dx.doi.org/10.1086/502044

[5] Lanbeck, P., Odenholt, I. and Paulsen, O. (2002) Antibiotics differ in their tendency to cause infusion phlebitis: A prospective observational study. Scandinavian Journal of Infectious Diseases, 34, 512-519. http://dx.doi.org/10.1080/0036554011008090

[6] Tagalakis, V., Kahn, S.R., Libman, M. and Blostein, M. (2002) The epidemiology of peripheral vein infusion thrombophlebitis: A critical review. American Journal of Medicine, 113, 146-151.

http://dx.doi.org/10.1016/S0002-9343(02)01163-4

[7] Higginson, R. and Parry, A. (2011) Phlebitis: Treatment, care and prevention. Nursing Times, 107, 18-21.

[8] Tagalakis, V., Kahn, S.R., Correa, J.A., et al. (2007) Thrombophilia in short peripheral catheter thrombophlebitis. Thrombosis Research, 119, 587-592. http://dx.doi.org/10.1016/j.thromres.2006.04.012

[9] Lanbeck, P., Odenholt, I. and Riesbeck, K. (2004) Diclo- 
xacillin and erythromycin at high concentrations increase ICAM-1 expression by endothelial cells: A possible factor in the pathogenesis of infusion phlebitis. Journal of Antimicrobial Chemotherapy, 53, 174-179. http://dx.doi.org/10.1093/jac/dkh056

[10] Lewis, G.B. and Hecker, J.F. (1985) Infusion thrombophlebitis. British Journal of Anaesthesia, 57, 220-233. http://dx.doi.org/10.1093/bja/57.2.220

[11] Subrahmanyam, M. (1989) Infusion thrombophlebitisHistological and bacteriological study. Indian Journal of Medical Sciences, 43, 231-234.

[12] Wagner, D.D. and Frenette, P.S. (2008) The vessel wall and its interactions. Blood, 111, 5271-5281. http://dx.doi.org/10.1182/blood-2008-01-078204

[13] Jesty, J., Yin, W., Perrotta, P. and Bluestein, D. (2003) Platelet activation in a circulating flow loop: Combined effects of shear stress and exposure time. Platelets, 14, 143-149. http://dx.doi.org/10.1080/0953710031000092839

[14] Albayrak, R., Yuksel, S., Colbay, M., et al. (2007) Hemodynamic changes in the cephalic vein of patients with hemodialysis arteriovenous fistula. Journal of Clinical Ultrasound: JCU, 35, 133-137.

[15] Kamm, R.D. and Pedley, T.J. (1989) Flow in collapsible tubes: A brief review. Journal of Biomechanical Engineering, 111, 177-179. http://dx.doi.org/10.1115/1.3168362

[16] Yaniv, S., Halpern, P., Aladgem, D., Zaretsky, U. and Elad, D. (2000) In vitro model of intravenous fluid administration: Analysis of vein resistance to rapid fluid delivery. Medical Engineering \& Physics, 22, 395-404. http://dx.doi.org/10.1016/S1350-4533(00)00046-1

[17] Henry, W.R., William, E.M. and Eugene, B.F.J. (1944) The influence of the collapsibility of veins on venous pressure, including a new procedure for measuring tissue pressure. Journal of Clinical Investigation, 23, 333-341. http://dx.doi.org/10.1172/JCI101499

[18] Goel, M.S. and Diamond, S.L. (2002) Adhesion of normal erythrocytes at depressed venous shear rates to activated neutrophils, activated platelets, and fibrin polymerized from plasma. Blood, 100, 3797-3803. http://dx.doi.org/10.1182/blood-2002-03-0712

[19] Michaux, G., Hewlett, L.J., Messenger, S.L., et al. (2003) Analysis of intracellular storage and regulated secretion of 3 von Willebrand disease-causing variants of von Willebrand factor. Blood, 102, 2452-2458. http://dx.doi.org/10.1182/blood-2003-02-0599

[20] Prasad Chennazhy, K. and Krishnan, L.K. (2005) Effect of passage number and matrix characteristics on differentiation of endothelial cells cultured for tissue engineering. Biomaterials, 26, 5658-5667. http://dx.doi.org/10.1016/j.biomaterials.2005.02.024

[21] Schwartz, E.A., Bizios, R., Medow, M.S. and Gerritsen, M.E. (1999) Exposure of human vascular endothelial cells to sustained hydrostatic pressure stimulates proliferation. Involvement of the alphaV integrins. Circulation Research, 84, 315-322. http://dx.doi.org/10.1161/01.RES.84.3.315

[22] O'Grady, N.P., Alexander, M., Burns, L.A., et al. (2011) Guidelines for the prevention of intravascular catheterrelated infections. American Journal of Infection Control, 39, S1-S34. http://dx.doi.org/10.1016/j.ajic.2011.01.003

[23] Yamakuchi, M., Kirkiles-Smith, N.C., Ferlito, M., et al. (2007) Antibody to human leukocyte antigen triggers endothelial exocytosis. Proceedings of the National Academy of Sciences USA, 104, 1301-1306. http://dx.doi.org/10.1073/pnas.0602035104 